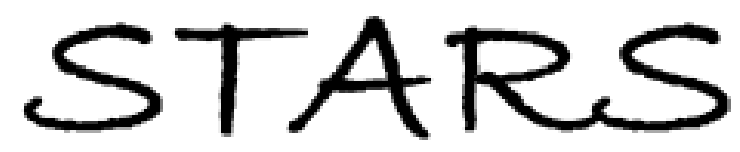

University of Central Florida

STARS

$1-1-2003$

\title{
Cathode ray tube addressed liquid crystal light valve projection display
}

Qiong-Hua Wang

University of Central Florida

Jianbo Cheng

Shin-Tson Wu

University of Central Florida

Changjun $\mathrm{Ge}$

Guangjiu Fang

See next page for additional authors

Find similar works at: https://stars.library.ucf.edu/facultybib2000

University of Central Florida Libraries http://library.ucf.edu

This Article is brought to you for free and open access by the Faculty Bibliography at STARS. It has been accepted for inclusion in Faculty Bibliography 2000s by an authorized administrator of STARS. For more information, please contactSTARS@ucf.edu.

\section{Recommended Citation}

Wang, Qiong-Hua; Cheng, Jianbo; Wu, Shin-Tson; Ge, Changjun; Fang, Guangjiu; Qi, Kangcheng; and Rao, Haibo, "Cathode ray tube addressed liquid crystal light valve projection display" (2003). Faculty Bibliography 2000s. 4111.

https://stars.library.ucf.edu/facultybib2000/4111

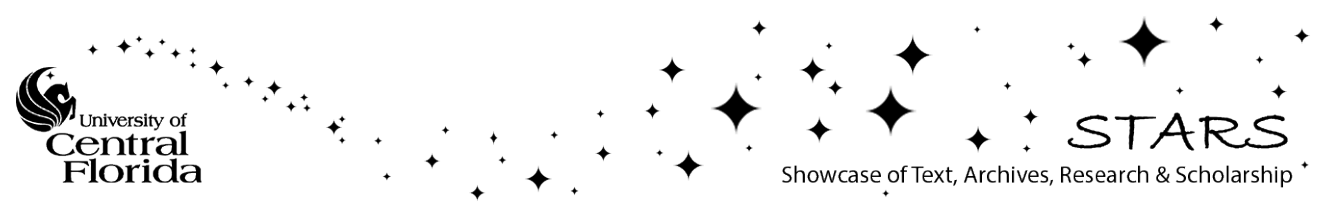




\section{Authors}

Qiong-Hua Wang, Jianbo Cheng, Shin-Tson Wu, Changjun Ge, Guangjiu Fang, Kangcheng Qi, and Haibo Rao 


\section{Cathode ray tube addressed liquid crystal light valve projection display}

\author{
Qiong-Hua Wang \\ University of Central Florida \\ CREOL/School of Optics \\ Orlando, Florida 32816-2700 \\ E-mail: qhwang@mail.ucf.edu \\ Jianbo Cheng \\ University of Electronic Science and \\ Technology \\ School of Opto-Electronic Information \\ Chengdu, 610054, China \\ Shin-Tson Wu \\ University of Central Florida \\ CREOL/School of Optics \\ Orlando, Florida 32816-2700 \\ Changjun Ge \\ Guangjiu Fang \\ Kangcheng Qi \\ Haibo Rao \\ University of Electronic Science and \\ Technology \\ School of Opto-Electronic Information \\ Chengdu, 610054, China
}

\section{Introduction}

Projection is an important technology for achieving large screen displays. Both transmissive and reflective projection systems have been developed. ${ }^{1,2}$ On the optical engines, digital light processing using micromirrors, ${ }^{3}$ cathode ray tube (CRT) addressed liquid crystal light valves (LCLVs), ${ }^{4,5}$ polysilicon thin-film-transistor liquid crystal displays, ${ }^{6}$ and liquid crystal on silicon $(\mathrm{LCoS})^{7,12}$ are potential candidates. Each technology has its own pros and cons and unique market.

The CRT-addressed LCLV has been demonstrated for high brightness and large screen displays. ${ }^{4,5}$ It exhibits vivid colors and film-like picture quality. However, the current CRT-addressed LCLV projector still has insufficient resolution. For example, the Hughes-JVC ILA $^{\circledR}$ projector $^{5}$ has a resolution of $2000 \mathrm{TV}$ lines, which is still inadequate for the electronic cinema. ${ }^{8}$

The epitaxy-grown monocrystalline garnet CRT phosphor screen exhibits a much higher resolution than the conventional one fabricated by depositing phosphor powder on a glass faceplate, except that its overall light efficiency is lower because of the waveguiding effect. Recently, the fabrication process and performance of the chrome- $(\mathrm{Cr})$ doped yttrium aluminum garnet (YAG) green phosphor screen have reached a satisfactory level. However, the blue and red phosphor screens still need improvement. ${ }^{9}$ Another technical difficulty is to scale the monocrystalline YAG phosphor screen up 4 to 5 inches. Therefore, the epitaxially grown monocrystalline garnet CRT is not suitable for direct view and full-color projection displays. ${ }^{10}$
We integrate the epitaxially grown monocrystalline garnet CRT's advantage-high resolution, and the LCLV's advantage - high brightness and large screen, to develop a compact CRT-addressed LCLV projection display system.

\section{Structure, Principle, and Performance}

The schematic diagram of the CRT-addressed LCLV projection display system is shown in Fig. 1. The three CRTs used can have the same phosphor screen color because they are used to address the photoconductive amorphous silicon $(\alpha$-Si) LCLVs rather than being used for high brightness projection display directly. High resolution is more important than high brightness. On the LCLV side, sandwiched between two transparent indium-tin oxide (ITO) electrodes are a layer of photoconductor, a light blocking layer, a dielectric mirror, and a liquid crystal layer.

In the $\alpha$-Si LCLVs employing nematic liquid crystals, ac voltage is applied across the transparent ITO electrodes. When there is no light illuminating the photoconductor, no voltage drops across the LC layer due to the high impedance of the photoconductor. When the photoconductor is exposed to CRT whose wavelength is within the bandgap, the photoactivated carriers reduce the impedance of the photoconductor and cause a spatial voltage pattern across the high impedance LC layer. A higher intensity image causes a larger voltage drop. If this voltage exceeds the threshold of the LC, the directors are reoriented along the electric field direction, resulting in phase retardation on the readout light. The analyzer transforms this phase modula- 
Wang et al.: Cathode ray tube ...

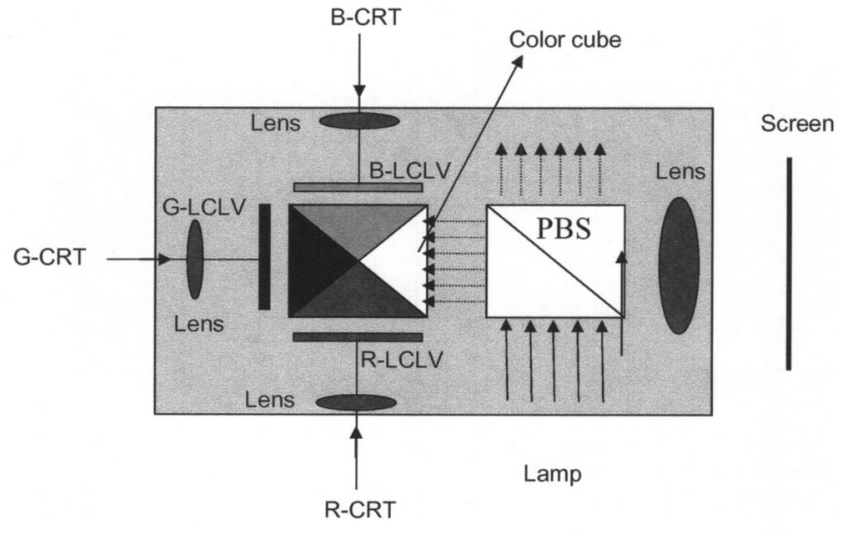

Fig. 1 The CRT-addressed LCLV projection display system.

tion into intensity modulation and completes the image conversion.

A typical frame (rise + decay) time for a $\alpha$-Si LCLV and $4-\mu \mathrm{m}$ LC cell gap is about $30 \mathrm{~ms}$ at room temperature. We have developed a compact optical system, including xenon arc lamp, a color cube and polarizer beam separator (PBS), projection lens, and projection screen.

\section{$2.1 \quad C R T$}

In a conventional CRT, phosphor powders are deposited on the glass substrate. But in Fig. 1 the CRT's phosphor screen is a Cr:YAG luminescent screen fabricated by doping $\mathrm{Cr}$ into a YAG substrate using a liquid phase epitaxy process. We have developed a new electron-vacuum glass that matches the YAG to make the CRT envelope. In addition, we have developed the seal between the YAG luminescent screen and the glass envelope using a new low temperature frit glass. A high resolution electron gun was designed for the CRT.

From a simple estimation the green Cr:YAG CRT needs $\sim 60 \mathrm{~cd} / \mathrm{m}^{2}$ luminance to fully activate the LCLV. Our Cr:YAG screen luminance is $159 \mathrm{~cd} / \mathrm{m}^{2}$ at a current of 0.1 $\mathrm{mA}$, which is sufficient to activate the LCLV.

The major advantage of the Cr:YAG luminescent screen over the conventional CRT is its high resolution. In a conventional CRT, the phosphor particles scatter the light around. As a result, the CRT resolution is somewhat smeared. In the monocrystalline Cr:YAG screen, the phosphor screen's resolution is as good as the spot size of the electron beam. The resolution of our Cr:YAG CRT is 2500 TV lines, which is much higher than that of a conventional CRT.

\section{$2.2 L C L V$}

The operating principle of the LCLV in Fig. 1 is similar to that described in Ref. 5. We deposited $\alpha$-Si:H and $\alpha-\mathrm{C}: \mathrm{H}$ in a plasma-enhanced chemical vapor deposition (PECVD) system as a photoconductor and light blocking layer, respectively. The dielectric mirror $\mathrm{TiO}_{2} / \mathrm{SiO}_{2}$ layers are fabricated by electron beam heating evaporation. To satisfy the requirement of high brightness, high resolution and contrast, and low operating voltage, we chose to use the 45-deg twisted nematic (TN) reflective liquid crystal cell. ${ }^{11}$ As shown in Fig. 1, three LCLVs are used with each addressed by a CRT. The 45-deg TN cell displays a normally black mode. To obtain a good dark state, each LCLV is biased at a slightly different voltage.

\subsection{Optical System}

The optical system architecture for the CRT-addressed LCLV is shown in Fig. 1. A 1-kW xenon arc lamp was used for illumination. The incident light is polarized and reflected by the polarizing beamsplitter toward a color cube. The color cube separates the polarized beam into three primary colors, and then reflects each color to a LCLV. The modulated light from each LCLV is reflected to the color cube. The color cube recombines them and projects the images to a screen through a projection lens. The layout of the optical system is reasonably compact.

\section{Performances}

We have built a CRT-addressed LCLV projection display prototype as shown in Fig. 1. With a 1-kW xenon lamp, the projector has $\sim 3000$ lumens light output, 2500 lines of resolution, and 1000:1 contrast ratio. To recover the lost $50 \%$ light from the PBS, one could implement a polarization conversion device. The PBS array polarization conversion device developed by Seiko-Epson is particularly interesting. ${ }^{6}$ A light recycling efficiency as high as $70 \%$ has been demonstrated.

\section{Conclusion}

We have developed a new Cr:YAG CRT for addressing the LCLV projector. The green Cr:YAG CRTs exhibit $2500 \mathrm{TV}$ lines of resolution and sufficient luminance for activating the amorphous silicon liquid crystal light valves. A compact projector prototype has been demonstrated. Such a projection system design holds promise for future large screen HDTV sets and electronic cinemas.

\section{References}

1. E. H. Stupp and M. S. Brennesholtz, Projection Displays, John Wiley and Sons, New York (1999).

2. S. T. Wu and D. K. Yang, Reflective Liquid Crystal Displays, WileySID, Chichester (2001).

3. F. Tatsumi, S. Moriya, and Y. Ishizaka, "Optical system using 3 Pieces of D-ILA panel module," Proc. Intl. Display Workshops, pp. 753-756 (1998).

4. V. J. Fritz, "Full-color liquid crystal light valve projector for shipboard use," Proc. SPIE 1255, 59-68 (1990).

5. R. D. Sterling and W. P. Bleha, "Electronic cinema using ILA® projector technology," SID Tech. Digest 30, 216-219 (1999).

6. N. Okamoto, "Developments in p-Si TFT LCD projectors," SID Tech. Digest 32, 1176-1179 (2001).

7. D. Armitage, "Silicon-chip liquid-crystal display," Proc. SPIE 2407, 280-290 (1995)

8. L. J. Thorpe, "HDTV and film-digitization and extended range," SMPTE J., 486 (1993)

9. J. M. Robertson and M. W. van Tol, "Epitaxially grown monocrystalline garnet cathode ray tube phosphor screens," Appl. Phys. Lett. 37, 471-473 (1980).

10. Q. H. Wang, J. B. Cheng, and Z. L. Lin, "A new YAG phosphor screen for projection CRT," Electron. Lett. 34, 420-1421 (1998).

11. J. Grinberg, A. Jacobson, W. Bleha, L. Miller, I. Fraas, D. Bosewell, 
Wang et al.: Cathode ray tube ...

and G. Myer, "A new real-time noncoherent light image converter: the hybrid field effect liquid crystal light valve," Opt. Eng. 14, 217 (1975).

12. M. Bone, "Front-projection optical-system design for reflective LCoS technology," J. Soc. Inf. Disp. 9, 213-218 (2001).

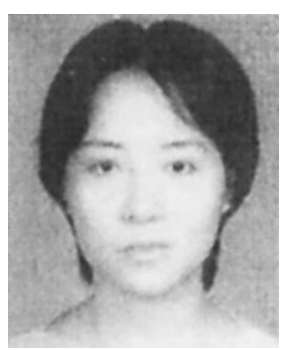

Qiong-Hua Wang is a post-doctorate research fellow in the School of Optics/ CREOL, University of Central Florida, and an associate professor in the School of Opto-Electronic Information, University of Electronic Science and Technology of China (UESTC). She received her MS and $\mathrm{PhD}$ degrees in opto-electronics and optical engineering from UESTC in 1995 and 2001, respectively. She has coauthored more than 20 papers on display devices and systems. Her recent research interests include CRT projection displays and liquid crystal displays.

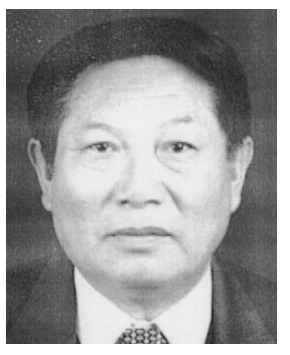

Jianbo Cheng is a professor of optoelectronics and optical engineering at the School of Opto-Electronic Information, University of Electronic Science and Technology of China (UESTC). He conducts teaching and research in the areas of $\mathrm{LaB}_{6}$ cathode, silicon target vidicon, YAG projection CRT, and TV and liquid crystal displays. He is the author of 40 technical papers and he has several patents to his credit. He won several national science and technology prizes. He is the recipient of the Distinguished Leadership Award proclaimed by the International Directory of Distinguished Leadership (seventh edition) for his outstanding contribution to contemporary society. He is a fellow of the Vacuum Society and the Society of Vacuum Electronics of China. Recently his research interests have focused on YAG projection CRT and TV, liquid crystal displays, organic light-emitting diodes display, and microlasers.

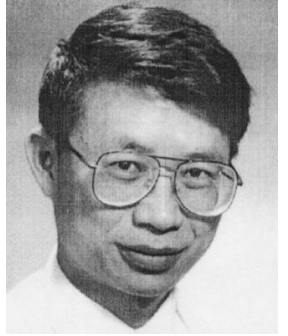

Shin-Tson Wu is a provost-distinguished professor of optics, School of Optics/ CREOL, University of Central Florida (UCF). He is a fellow of SID and OSA, and a recipient of the SID special recognition award, ERSO special achievement award, the Hughes team achievement award, and the Hughes annual outstanding paper award. He has coauthored two books, three book chapters, and has published more than 150 journal papers. His studies at UCF concentrate in five areas: nanophotonics, optical phased arrays for laser beam steering, high birefringence liquid crystals, liquid crystal TVs and computer monitors and transflective displays, and bioinspired optics.

Changjun Ge is a technical director of Shanghai Novel CPT Incorporated, China. He received his MS and PhD degrees in optoelectronics and optical engineering from the University of Electronic Science and Technology of China in 1992, and 2000, respectively.

Guanjiu Fang is a PhD candidate in physics at the University of Colorado. He received the MS degree in opto-electronics from the University of Electronic Science and Technology of China in 1995.

Kangcheng Qi is an associate professor at the School of OptoElectronic Information, University of Electronic Science and Technology of China (UESTC). He received the MS degree in optoelectronics and optical engineering from UESTC in 1995. His research interests include CRT projection display and liquid crystal display.

Haibo Rao is an associate professor at the School of OptoElectronic Information, University of Electronic Science and Technology of China (UESTC). He received the MS and PhD degrees in physics and opto-electronics from Sichuan University and UESTC in 1990, and 2000, respectively. His research interests include CRT projection display and microlasers. 